\title{
Is Juvenile Salmon Condition Driven by the Nutritional Quality at the Base of the Plankton Food-web?
}

\author{
David Costalago ${ }^{1}$, Brian P. V. Hunt ${ }^{1,2,3}$, Ian Perry ${ }^{4}$, Chrys Neville ${ }^{4}$, Kelly Young ${ }^{5}$, and Ian Forster ${ }^{6}$ \\ ${ }^{l}$ Institute for the Oceans and Fisheries, University of British Columbia, Vancouver BC, Canada \\ ${ }^{2}$ Department of Earth, Ocean and Atmospheric Sciences, 2207 Main Mall, University of British Columbia, \\ Vancouver, BC, V6T1Z4, Canada \\ ${ }^{3}$ Hakai Institute, Heriot Bay BC, Canada \\ ${ }^{4}$ Pacific Biological Station, Fisheries and Oceans Canada, Nanaimo BC, Canada \\ ${ }^{5}$ Institute of Ocean Sciences, Fisheries and Oceans Canada, Sidney, BC, Canada \\ ${ }^{6}$ Centre for Aquaculture and Environmental Research, Fisheries and Oceans Canada, Vancouver BC, Canada
}

Keywords: Strait of Georgia, fatty acids, juvenile salmon

A first step towards understanding the impact of long-term changes in oceanographic conditions and zooplankton communities on salmon is characterizing the food-web pathways that support them. Salmon's juvenile phase includes their transition from freshwater to the ocean, and there is evidence pointing to the importance of nutritional condition during these early life stages as being critical for the success of the adults' return (Beamish and Mahnken 2001). The study of the food quality and plankton-based energy sources for Pacific salmon during the fish's early ocean phase offers information on how salmon condition relates to the environment. The Strait of Georgia (SoG) supports the early marine phase of salmon emanating from British Columbia's biggest salmon producer, the Fraser River, and most smolts in the region out-migrate between February-June.

We thus analyzed the regional and seasonal fatty acid (FA) composition dynamics of zooplankton and particulate organic matter (POM) in the SoG. Specifically, we quantified the FAs 20:5n3 (EPA) and 22:6n3 (DHA), which are essential for the fish development, and we calculated the DHA to EPA ratio (DHA:EPA), which is commonly used as a proxy for nutritional quality of food for fish (Kainz et al. 2004) and for the nutritional condition of the fish themselves. In particular, higher DHA:EPA values have been linked to relatively higher abundance of dinoflagellates compared to diatoms and better fish condition.

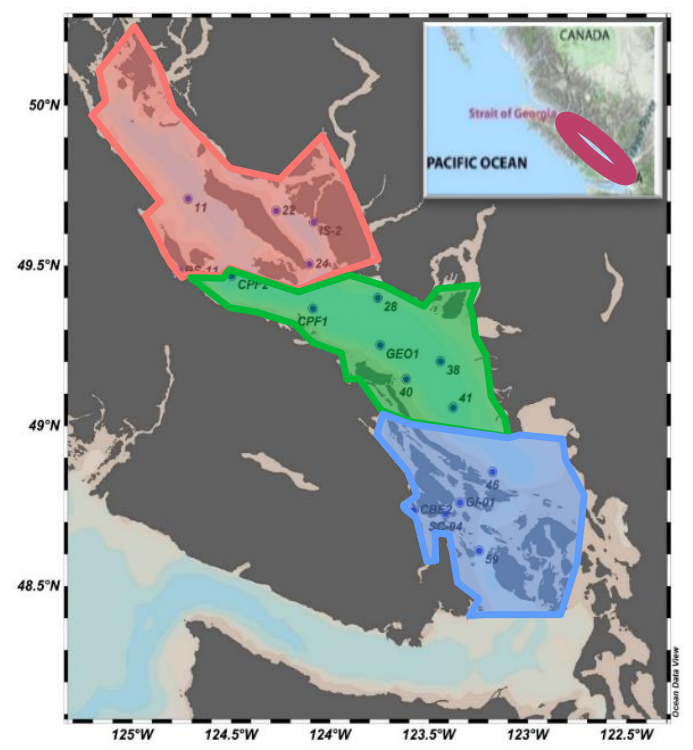

Fig. 1. Map of the Strait of Georgia with the sampling sites (blue points) and the three regions established for this study (red $=$ north, green $=$ central, blue $=$ south .

Methods

Particulate organic matter (POM) and zooplankton samples were collected between 2015-2018 by filtering 10 $\mathrm{L}$ of surface water through $47 \mathrm{~mm} \mathrm{GF/F}$ filters and with a pair of $64 \mu \mathrm{m}$ mesh-size bongo nets, respectively.

Immediately after collection, zooplankton were size-fractionated on board through a set of sieves and flash-frozen in liquid nitrogen. In the lab, we sorted the zooplankton samples according to the following size groups: small (64- 
$250 \mu \mathrm{m})$, medium $(500-1,000 \mu \mathrm{m})$ and large $(1,700-8,000 \mu \mathrm{m})$. Additional samples of large zooplankton were sorted by species for taxon-specific analyses.

We grouped samples according to their location in three main regions: North-north of $49.48^{\circ} \mathrm{N}$, where the influence from the Pacific Ocean and from the Fraser River are weak; Central-between $49.48^{\circ} \mathrm{N}$ (southern tip of Texada Island) and $49^{\circ} \mathrm{N}$ (southern part of the Fraser River Delta), which is the area receiving most of the influence from the Fraser River; and South — south of $49^{\circ} \mathrm{N}$, which comprises the Gulf Islands region and is subjected to a greater influence from the Pacific Ocean than the rest of the SoG (Fig. 1). Samples were prepared for fatty acid (FA) analysis as in Forster et al. (2011).

\section{Results and Discussion}

Potential prey (i.e., large zooplankton) for smolts have better nutritional quality in the southern SoG than in the northern region (Fig. 2), which suggests that fish that remain in the southern region for longer might benefit from the prey quality more than smolts that migrate north immediately after leaving the river.

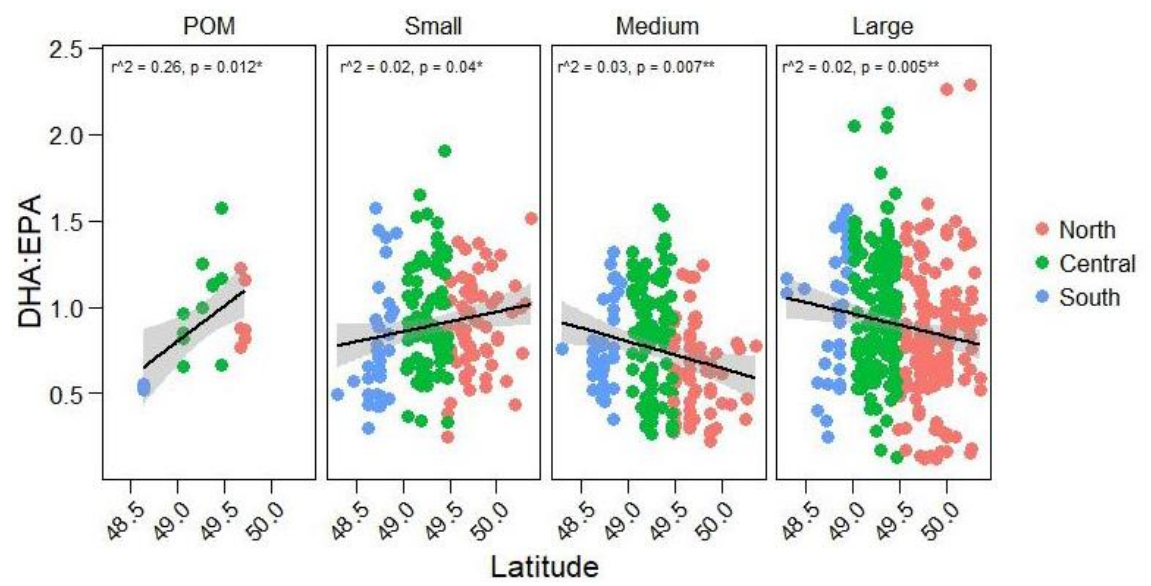

Fig. 2. DHA:EPA increases from south to north in POM and in small zooplankton, but decreases with latitude in medium and large zooplankton, which are the potential prey for juvenile salmon.

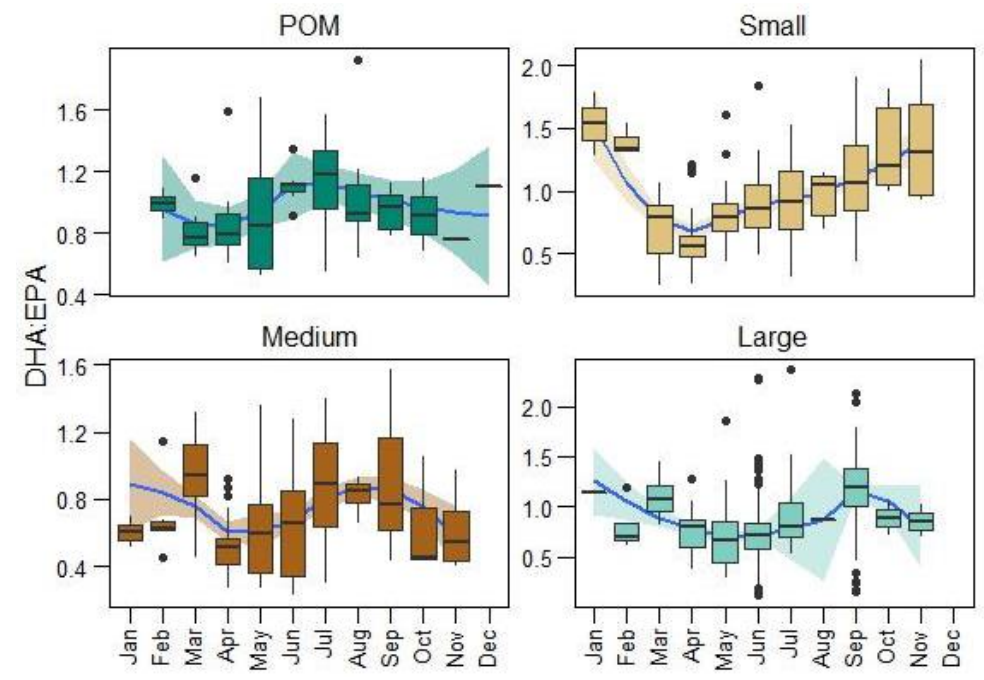

Fig. 3. DHA:EPA values were significantly lower in spring (April-June) than in summer (July-September) and winter (January-March) for POM and all zooplankton size fractions.

Regarding the seasonal patterns of plankton FAs we found that, for the period 2015-2018, DHA:EPA in POM was higher between May-August (Fig. 3). However, in all zooplankton size fractions DHA:EPA values were significantly lower in spring (April-June) than in summer (July-September) (Fig. 3). DHA:EPA > 1 indicates a dominance in the contribution of dinoflagellates, whereas a value $<1$ suggests a greater contribution of diatoms (Budge and Parrish 1998). Thus, our seasonal data of DHA:EPA in POM accurately captures the diatom-rich spring bloom in the SoG (Fig. 3). Therefore, smolts out-migrating later in the season (i.e., summer) might encounter better quality food than in spring. 


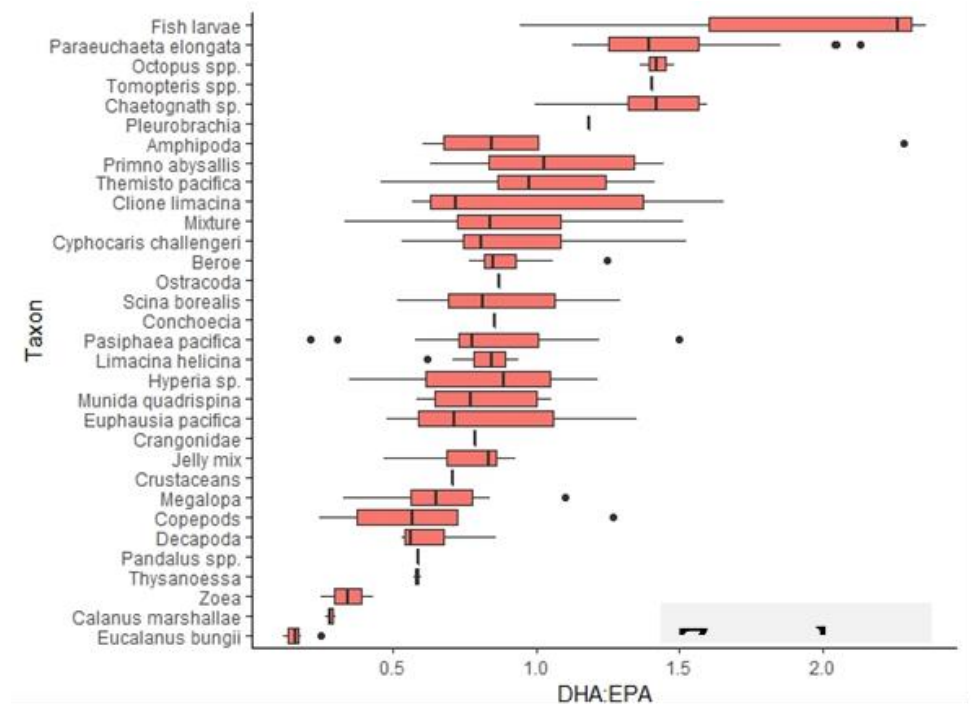

Fig. 4. Fish larvae, P. elongata, Octopus spp. and Tomopteris spp. are the potential salmon prey with the highest nutritional quality, whereas $C$. marshallae and E. bungii presented the lowest nutritional quality. All individuals were obtained from the large zooplankton size fraction (i.e., larger than $1,700 \mu \mathrm{m}$ ).

We also showed that fish larvae, chaetognaths, most amphipod species and the carnivorous copepod Paraeuchaeta elongata, among other groups, have higher DHA:EPA than POM, whereas other copepod species (e.g., Calanus marshallae and Eucalanus bungii), decapods and euphausiids have lower DHA:EPA (Fig. 4). This indicates that a diet based on species from the former group might translate to a better salmon juvenile nutritional condition compared to salmon that feed mostly on decapods and euphausiids. Consequently, we hypothesize that the decrease in the juvenile salmon condition might be explained by a disruption in the food-web pathways to juvenile salmon between POM and zooplankton.

\section{REFERENCES}

Beamish, R., and C. Mahnken. 2001. A critical size and period hypothesis to explain natural regulation of salmon abundance and the linkage to climate and climate change. Prog. Oceanogr. 49: 423-437. doi:10.1016/S00796611(01)00034-9.

Budge, S.M., and C.C. Parrish. 1998. Lipid biogeochemistry of plankton, settling matter and sediments in Trinity Bay, Newfoundland. II. Fatty acids. Org. Geochem. 29: 1547-1559. doi:10.1016/S0146-6380(98)00177-6.

Forster, I.P., W.G. Dominy, L.G. Obaldo, G.F. Harnell, E.F. Sanders, T.C. Hickman, and M.C. Ruebelt. 2011. The effect of soybean oil containing stearidonic acid on growth performance, n-3 fatty acid deposition and sensory characteristics of pacific white shrimp (Litopenaeus vannamei). Aquacult. Nutr. 17: 200-213. doi:10.1111/j.1365-2095.2009.00726.x.

Kainz, M., M.T. Arts, and A. Mazumder. 2004. Essential fatty acids in the planktonic food web and their ecological role for higher trophic levels. Limnol. Oceanogr. 49: 1784-1793. doi:10.4319/lo.2004.49.5.1784. 